\title{
ARTIGO TÉCNICO \\ AVALIAÇÃO DE MODELO MATEMÁTICO PARA ESTIMAR A RADIAÇÃO SOLAR INCIDENTE SOBRE SUPERFÍCIES COM DIFERENTES EXPOSIÇÕES E DECLIVIDADES
}

\author{
JOSÉ E. P. TURCO ${ }^{1}$, GILCILEIA S. RIZZATTI ${ }^{2}$
}

RESUMO: Este trabalho teve por objetivo avaliar modelo matemático para estimar a radiação solar global diária sobre superfícies com diferentes exposições e declividades, no período de março de 2002 a março de 2003. A pesquisa foi desenvolvida em uma estrutura denominada "Bacia Hidrográfica Experimental" do Departamento de Engenharia Rural da UNESP, Câmpus de Jaboticabal - SP. Nessa estrutura, foram utilizadas superfícies caracterizadas como H, 10N, 10S, 20N, 20S, 10E, 10W, 20E e 20W. O sensor utilizado para medir a radiação solar global incidente nas superfícies estudadas foi um piranômetro da marca Kipp \& Zonnen, modelo CM3. Para calcular a radiação solar incidente nas superfícies estudadas, foi utilizado o modelo de Kondratyev. As análises dos resultados foram feitas para o período diário, utilizando-se de análise de regressão e considerando o modelo linear $(y=a x+$ b), na qual a variável dependente foi a radiação global medida $(\mathrm{K} \downarrow \mathrm{M})$ e a radiação global calculada $(\mathrm{K} \downarrow \mathrm{C})$ foi a variável independente. Os resultados deste estudo mostram que o modelo apresentou bons resultados para estimar a radiação nas superfícies H, 10N, 10S, 10E, 10W, 20E e 20W. Utilizando-se de dados de dias com céu límpido, foram obtidos os seguintes resultados: no inverno, o modelo foi preciso para estimar a radiação solar na superfície $20 \mathrm{~N}$, e apresentou resultados aceitáveis para estimar a radiação solar na superfície 20 S.

PALAVRAS-CHAVE: modelo, radiação global, superfícies inclinadas.

\section{EVALUATION OF A MODEL USED FOR ESTIMATING INCOME SOLAR RADIATION IN SURFACES WITH DIFFERENT EXPOSITIONS AND DECLIVITIES}

\begin{abstract}
This research evaluated a model used for estimating income solar radiation in surfaces with different expositions and declivities. The research was developed in one structure denominated "Experimental Hidrografic Basin" of the Rural Engineering Department - UNESP, Sao Paulo State University, Brazil. In this structure was utilized surfaces characterized as H, 10N, 10S, 20N, 20S, 10E, 10W, 20E and 20W. The sensor used for obtaining income solar radiation in surfaces was a Kipp \& Zonnen Piranometer model CM3. The methodology of Kondratyev was used to calculate the income solar radiation on surfaces. Evaluation of the results were made for the daily period using regression analyze and considering the linear model $(y=a x+b)$, which the dependent variable was the global radiation measured $(\mathrm{K} \downarrow \mathrm{M})$ and the global radiation calculated $(\mathrm{K} \downarrow \mathrm{C})$ was the independent variable. The model showed good results to estimate the radiation on the surfaces $\mathrm{H}, 10 \mathrm{~N}, 10 \mathrm{~S}, 10 \mathrm{E}, 10 \mathrm{~W}, 20 \mathrm{E}$ e $20 \mathrm{~W}$. Using data of sunny days were obtained the following results: in the winter, the model was exact to estimate the solar radiation on the surface $20 \mathrm{~N}$, and it showed acceptable results to estimate the solar radiation on the surface $20 \mathrm{~S}$.
\end{abstract}

KEYWORDS: model, global radiation, inclined surfaces.

\footnotetext{
${ }_{1}^{1}$ Prof. Adjunto, Departamento de Engenharia Rural, UNESP, Jaboticabal - SP, Fone: (0XX16) 3209.2637, jepturco@ fcav.unesp.br

${ }^{2}$ Eng ${ }^{\mathrm{a}}$ Agrônoma, Mestranda em Ciência do Solo, UNESP, Jaboticabal - SP.

Recebido pelo Conselho Editorial em: 30-11-2004

Aprovado pelo Conselho Editorial em: 13-2-2006 


\section{INTRODUÇÃO}

O conhecimento da irradiância solar sobre superfícies que não sejam horizontais, é requerido em grande variedade de aplicações, principalmente no setor agrícola.

Superfícies com diferentes exposições e declividades recebem diferentes totais de radiação solar, a qual é o fator primário que condiciona os elementos climatológicos e fisiológicos relacionados à produtividade.

Medidas de irradiância solar em superfícies com diferentes exposições e declividades são muito onerosas. Uma das principais causas disso é o alto custo dos sensores para tais medidas, o que estimula a elaboração de modelos que permitam estimar, a partir de determinações em superfície horizontal, a irradiância em terrenos com diferentes exposições e declividades.

Um modelo matemático muito utilizado para estimar a radiação solar global em superfícies com diferentes exposições e declividades, a partir de determinações na horizontal, é o de KONDRATYEV (1977). Nesse sentido, torna-se relevante a avaliação desse modelo para verificar a sua precisão.

A radiação solar que atinge a superfície terrestre é constituída da radiação solar direta e radiação solar difusa. A radiação direta é a parcela da energia radiante que chega diretamente na superfície do solo, e a radiação difusa é a outra parcela de energia radiante proveniente das demais direções que, em dias de céu limpo, atinge apenas $15 \%$ do total da radiação que chega à superfície terrestre. A energia radiante total na superfície terrestre, que é a soma da radiação direta e difusa, é a radiação solar global (VIENELLO \& ALVES, 1991).

A quantidade total de radiação recebida na superfície de uma rampa varia de acordo com a exposição e inclinação, sendo sua componente direta influenciada por ambas e a sua componente difusa apenas pela inclinação, segundo CHANG (1968), de forma que, em dias nublados, o efeito da exposição é minimizado.

KONDRATYEV \& MANOLOVA (1960), baseados em modelos teóricos e usando dados obtidos em piranômetros montados em teodolitos, estimaram as componentes do balanço de radiação para 37 direções e ângulos de $15 ; 40$ e $65^{\circ} \mathrm{com}$ a vertical, a cada $30^{\circ}$ de azimute e no zênite, sob céu limpo e nublado, e verificaram que, para inclinações até $10^{\circ}$ (declividades até $17,6 \%$ ), a orientação tem pouca influência na variação da radiação total, sendo, porém, levemente diferente da horizontal; acima de $10^{\circ}$, depende essencialmente da orientação e declividade. Finalmente, estabeleceram que, para Criméia, $45^{\circ} \mathrm{N}$ de latitude, onde se realizaram as medidas, a radiação direta é a principal componente diferencial de orientações e declividades.

LATANZE et al. (1987) utilizaram o modelo de KONDRATYEV (1977) para a estimativa da radiação solar incidente sobre superfícies com diferentes orientações em Jaboticabal - SP. Verificaram que os totais de radiação solar medidos em diferentes orientações, em diferentes épocas do ano, apresentaram diferenças inferiores a $2 \%$ comparados com aqueles estimados pela metodologia proposta.

ARAGON \& TOLEDO PIZA (1991) construíram um modelo teórico para o cálculo da energia solar total diária incidente em rampas planas com diferentes inclinações e exposições, e obtiveram resultados satisfatórios comparando os valores teóricos com os experimentais.

COLLE \& PEREIRA (1998) fizeram parte da equipe que desenvolveu um modelo computacional denominado BRAZILSR, capaz de determinar a intensidade da radiação solar incidente na superfície, a partir de imagens de satélite. A validação do modelo foi realizada comparando-se as estimativas do modelo com os dados de piranômetros de primeira linha disponíveis em várias localidades, no território nacional. 
KANG et al. (2002) desenvolveram um modelo denominado TopoRad, que possibilita obter amostras espaciais e temporais da radiação solar baseado na topografia e temperatura diária. O modelo foi aplicado na Floresta de Jumbong, localizada na Coréia do Sul. Concluíram que TopoRad apresentou bons resultados para predizer radiação solar diária em escala reduzida.

A finalidade deste trabalho foi avaliar um modelo matemático para estimativa da radiação solar global incidente sobre superfícies com diferentes exposições e declividades.

\section{MATERIAL E MÉTODOS}

A pesquisa foi desenvolvida em área experimental do Departamento de Engenharia Rural da UNESP, Câmpus de Jaboticabal - SP, situada a $21^{\circ} 15$ '22" de latitude sul, $48^{\circ} 18^{\prime} 58^{\prime \prime}$ de longitude oeste e altitude de 575 m, em uma estrutura denominada "Bacias Hidrográficas Experimentais", descrita com detalhes por LOPES (1986). O clima, de acordo com a classificação de Köeppen, é do tipo Cwa.

Nessa estrutura, foi realizado experimento no período de março de 2002 a março de 2003, na qual se utilizaram superfícies de $10,5 \mathrm{~m}^{2}$, caracterizadas como $\mathrm{H}$ (horizontal), $10 \mathrm{~N}(10 \%$ de declividade e exposição norte), $20 \mathrm{~N}$ (20\% de declividade e exposição norte), $10 \mathrm{~S}$ (10\% de declividade e exposição sul), 20S (20\% de declividade e exposição sul), 10E (10\% de declividade e exposição leste), 20E (20\% de declividade e exposição leste), 10W (10\% de declividade e exposição oeste) e 20W (20\% de declividade e exposição oeste), que simulam terrenos com exposições e declividades comumente utilizados na agricultura.

Nas superfícies da área experimental, foi plantada a grama batatais (Paspalum notatum Flügge) para simular as condições normalmente utilizadas em um Posto Meteorológico. Nas superfícies, foram efetuadas irrigações para manter o solo em boas condições hídricas.

Para a determinação da radiação solar global incidente nas superfícies, foi instalado, no centro da área de cada superfície, um piranômetro (KIPP \& ZONNEN) modelo CM3, paralelo à superfície, totalizando nove equipamentos. O sensor de cada superfície foi fixado em uma estrutura de alumínio, que o manteve a 1,0 $\mathrm{m}$ acima da superfície.

Os dados de radiação solar global incidente nas superfícies foram registrados por um sistema de aquisição de dados, composto por um Datalogger CR10 da marca Campbell Scientific, Inc., sendo instalado um sistema de aquisição de dados em cada bacia.

Para calcular a radiação solar nas superfícies estudadas, a partir da medida na horizontal, foi utilizada a metodologia desenvolvida por KONDRATYEV (1977), descrita a seguir:

O fluxo de radiação solar direta sobre uma superfície inclinada, com orientação arbitrária $\left(\mathrm{S}_{\mathrm{S}}\right)$, pode ser expresso por meio do fluxo de radiação recebido por uma superfície normal aos raios solares, usando a equação: em que,

$\mathrm{S}_{\mathrm{S}}=\mathrm{S}_{\mathrm{m}} \cos \mathrm{i}$

$\mathrm{S}_{\mathrm{m}}$ - fluxo de radiação solar recebido por uma superfície normal aos raios solares com presença da massa atmosférica $(\mathrm{m})$, e

i - ângulo de incidência dos raios solares numa determinada superfície inclinada.

O cosseno do ângulo de incidência dos raios é determinado pela eq.(2):

$$
\operatorname{cosi}=\cos \alpha \operatorname{senh}+\operatorname{sen} \alpha \cosh \cos \psi
$$


em que,

$\alpha$ - ângulo de inclinação da rampa em relação à horizontal;

$\mathrm{h}$ - ângulo de elevação solar;

$\psi-\psi \phi-\psi n$

$\omega \phi$ - azimute do sol, e

$\psi n$ - azimute da projeção da normal à rampa.

Os ângulos de elevação e de azimute do sol são determinados pelas equações:

$$
\begin{aligned}
& \operatorname{senh}=\operatorname{sen} \phi \operatorname{sen} \delta+\cos \phi \cos \delta \cos \Omega \\
& \cos \psi_{\theta}=\frac{\operatorname{senh} \operatorname{sen} \phi-\operatorname{sen} \delta}{\cosh \cos \phi} \\
& \operatorname{sen} \psi_{\theta}=\frac{\cos \psi \operatorname{sen} \Omega}{\cosh }
\end{aligned}
$$

em que,

$\phi$ - latitude do local;

$\delta$ - declinação do sol, e

$\Omega$ - ângulo horário do sol em dado instante.

Considerando as eqs.(2) a (5), a eq.(1) poderá ser escrita da seguinte maneira:

$$
\mathrm{Ss}=\operatorname{Sm}\{\cos \alpha \operatorname{senh}+\operatorname{sen} \alpha[\cos \psi n(\operatorname{tg} \varphi \operatorname{senh}-\operatorname{sen} \delta \sec \phi)+\operatorname{sen} \psi n \cos \delta \operatorname{sen} \Omega]\}
$$

A eq.(6) é a expressão geral para o cálculo da radiação solar instantânea recebida por terrenos com exposição determinada pelos ângulos $\alpha$ e $\psi$ n, para qualquer latitude “ $\phi$ ", em qualquer momento do dia " $\Omega$ " e em qualquer época do ano " $\delta$ ".

Para o caso particular da superfície horizontal $(\mathrm{H})$ do local $(\alpha=0)$, a eq.(6) pode ser escrita:

$$
\begin{aligned}
& \mathrm{SH}=\mathrm{S}_{\mathrm{m}} \operatorname{senh} \\
& \mathrm{Sm}=\frac{\mathrm{S}_{\mathrm{H}}}{\operatorname{sen} \phi \operatorname{sen} \delta+\cos \phi \cos \delta \cos \Omega}
\end{aligned}
$$

Para o cálculo da radiação solar incidente em rampas com qualquer exposição, num dado instante, pode-se usar a eq.(6), sob a seguinte forma:

$$
\begin{aligned}
& \mathrm{S}_{\mathrm{s}}=\frac{\mathrm{S}_{\mathrm{H}}}{(\operatorname{sen} \phi \operatorname{sen} \delta+\cos \phi \cos \delta \cos \Omega)} \cdot \\
& .\left\{\cos \alpha \operatorname{sen} \phi \operatorname{sen} \delta+\operatorname{sen} \alpha\left[\cos \psi_{\mathrm{n}}(\operatorname{tg} \varphi \operatorname{sen} \phi \operatorname{sen} \delta-\operatorname{sen} \delta \sec \varphi)\right]+\right. \\
& +\cos \Omega\left(\cos \alpha \cos \phi \cos \delta+\operatorname{sen} \alpha \cos \psi_{\mathrm{n}} \operatorname{sen} \phi \cos \delta\right)+ \\
& \left.+\operatorname{sen} \Omega \operatorname{sen} \alpha \cos \delta \operatorname{sen} \psi_{\mathrm{n}}\right\}
\end{aligned}
$$

ou, simplificando:

$$
\mathrm{S}_{\mathrm{s}}=\frac{\mathrm{S}_{\mathrm{H}}}{\operatorname{senh}}\left(\mathrm{A} 1+\mathrm{B} 1 \cos \Omega+\mathrm{C}_{1} \operatorname{sen} \Omega\right)
$$

em que, 


$$
\begin{aligned}
& \mathrm{A} 1=\cos \alpha \operatorname{sen} \phi \operatorname{sen} \delta+\operatorname{sen} \alpha\left[\cos \psi_{\mathrm{n}}(\operatorname{tg} \varphi \operatorname{sen} \phi \operatorname{sen} \delta-\operatorname{sen} \delta \sec \varphi)\right] \\
& \mathrm{B} 1=\cos \alpha \cos \phi \cos \delta+\operatorname{sen} \alpha \cos \psi_{\mathrm{n}} \operatorname{sen} \phi \cos \delta, \mathrm{e} \\
& \mathrm{C} 1=\operatorname{sen} \alpha \cos \delta \operatorname{sen} \psi_{\mathrm{n}} .
\end{aligned}
$$

As análises dos resultados foram feitas para o período diário, utilizando-se da análise de regressão e considerando o modelo linear $(y=a x+b)$, no qual a variável dependente foi a radiação solar global medida $(\mathrm{K} \downarrow \mathrm{M})$ e a radiação solar global calculada $(\mathrm{K} \downarrow \mathrm{C})$ foi a variável independente. Nessa análise, foi considerado o modelo completo, ou seja, $K \downarrow M=a \quad K \downarrow C+b$. Os valores calculados foram avaliados em relação aos medidos por meio da linha da igualdade perfeita $\left(45^{\circ}\right)$.

\section{RESULTADOS E DISCUSSÃO}

Por meio da análise de regressão linear, para as quatro estações do ano, pôde-se observar que o modelo estudado estimou com boa precisão a radiação solar global incidente nas superfícies H, 10N, $10 \mathrm{E}, 10 \mathrm{~W}, 20 \mathrm{E}$ e $20 \mathrm{~W}$, uma vez que os dados estavam em torno da reta 1:1. Verificou-se, também, boa distribuição dos pares de dados em torno da linha 1:1, confirmada pelos coeficientes de correlação, que variaram de 0,92 a 1 .

Para a primavera e o verão, o modelo apresentou o mesmo resultado citado para estimar a radiação global incidente nas superfícies $10 \mathrm{~S}, 20 \mathrm{~N}$ e $20 \mathrm{~S}$.

Pela Figura 1, para o outono e o inverno, pode-se observar que o modelo apresentou tendência em subestimar a radiação solar na superfície $10 \mathrm{~S}$, porém apresentando bom coeficiente para as duas estações do ano.
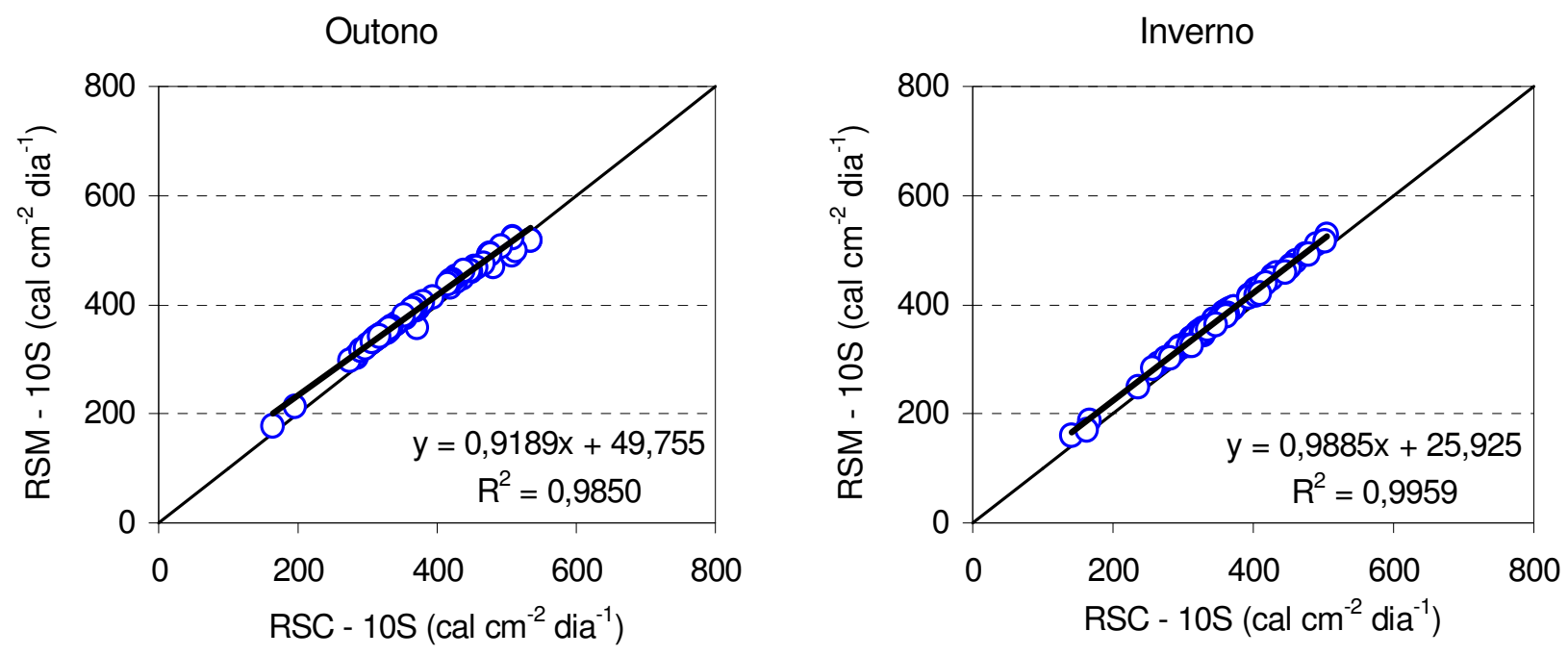

FIGURA 1. Correlação entre os valores diários da radiação solar global calculada em relação à radiação solar medida, na superfície 10S, para outono e inverno.

Na Figura 2, para a superfície 20N, no outono, há tendência de o modelo superestimar a radiação solar para valores inferiores à média e subestimar aqueles superiores.

Verifica-se, na Figura 3, para a superfície 20S, que, para o outono, a linha de tendência da radiação solar medida em relação à calculada apresenta subestimativa e tende a diminuir à medida que aumenta a radiação solar. Porém apresenta bom coeficiente de correlação $\left(\mathrm{R}^{2}=0,9631\right)$. 
Tais resultados podem ser considerados aceitáveis, tendo em vista que o modelo deixa de computar a radiação difusa, bem como aquela que o meio ambiente reflete para o instrumento.

Nota-se, também, nas Figuras 2 e 3, que, para o inverno, o modelo superestimou a radiação solar global incidente na superfície $20 \mathrm{~N}$ e subestimou na superfície $20 \mathrm{~S}$, bem como os pontos apresentaramse dispersos em torno da média, confirmados pelos valores dos coeficientes de correlação $\left(R^{2}=0,8843\right.$ e 0,8017$)$. Portanto, o modelo não apresentou bons resultados para estimar a radiação solar global incidente nas superfícies $20 \mathrm{~N}$ e $20 \mathrm{~S}$, para o inverno.

Outono

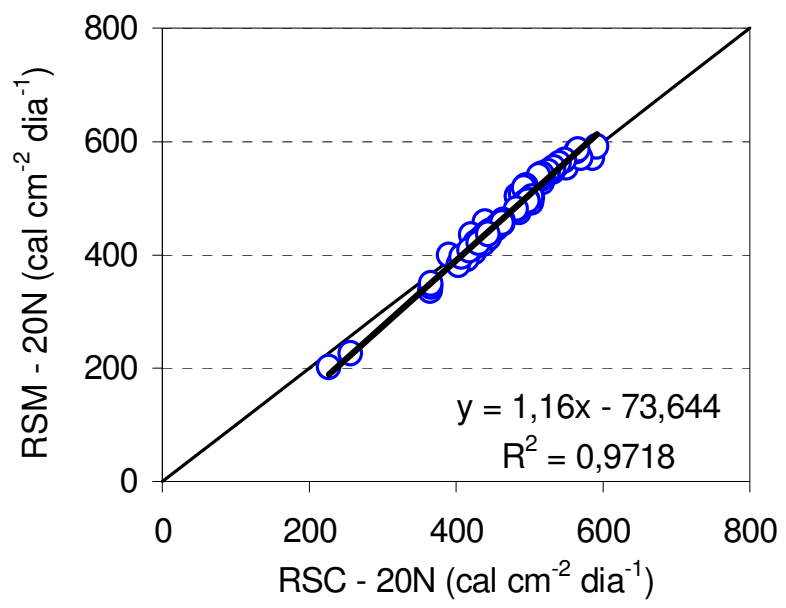

Inverno

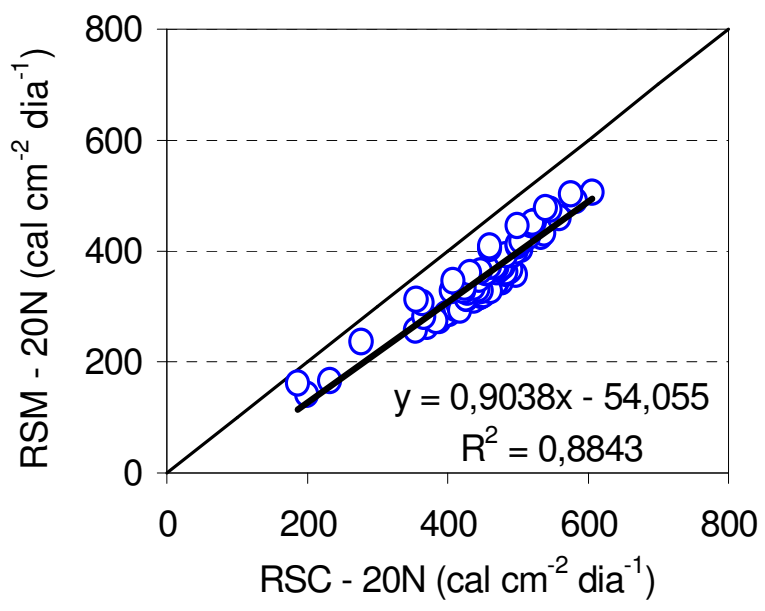

FIGURA 2. Correlação entre os valores diários da radiação solar global calculada em relação à radiação solar medida, na superfície $20 \mathrm{~N}$, para outono e inverno.

Outono

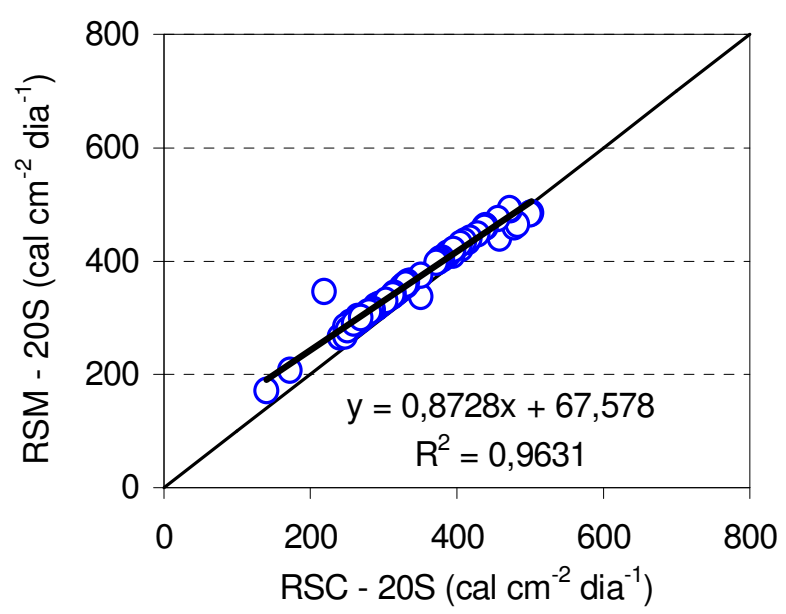

Inverno

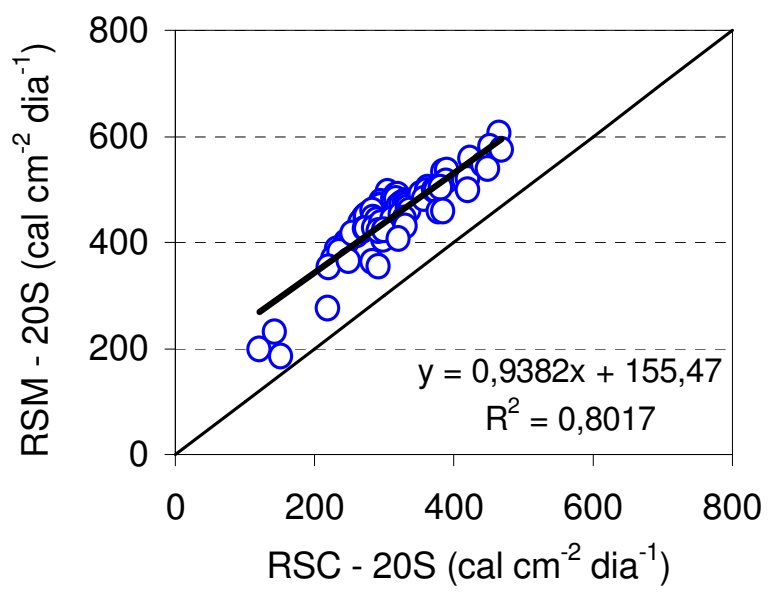

FIGURA 3. Correlação entre os valores diários da radiação solar global calculada em relação à radiação solar medida, na superfície $20 \mathrm{~S}$, para outono e inverno.

Para melhor avaliação do modelo estudado, foi feita, para as superfícies $10 \mathrm{~S}, 20 \mathrm{~N}$ e $20 \mathrm{~S}$, no outono e inverno, a correlação entre os valores diários da radiação solar global calculada em relação à radiação solar medida, para os dias com céu límpido (Figuras 4; 5 e 6). 
Verifica-se, na Figura 4, para o outono e inverno, e nas Figuras 5 e 6, para o outono, que, para as superfícies $10 \mathrm{~S}, 20 \mathrm{~N}$ e $20 \mathrm{~S}$, os resultados obtidos foram semelhantes aos obtidos para dias com céu nublado, com céu parcialmente nublado e com céu límpido.

Na Figura 5, para o inverno, pode-se observar que o modelo estudado estimou com boa precisão a radiação solar global incidente na superfície $20 \mathrm{~N}$, uma vez que os dados estão em torno da reta 1:1. Verifica-se, também, boa distribuição dos pares de dados em torno da linha 1:1, confirmada pelos coeficientes de correlação $\left(R^{2}=0,9956\right)$.

Na Figura 6, nota-se que, para o inverno, o modelo subestimou a radiação solar global incidente na superfície $20 \mathrm{~S}$, bem como os pontos estão em torno da média $\left(\mathrm{R}^{2}=0,9992\right)$. Portanto, o modelo apresentou resultados considerados aceitáveis para estimar a radiação solar global incidente na superfície 20S, para o inverno.

Nas Tabelas 1 e 2, são mostrados os valores diários medidos (RSM) e calculados (RSC) da radiação solar global incidente para as superfícies $20 \mathrm{~N}$ e $20 \mathrm{~S}$, juntamente com as diferenças percentuais entre os mesmos, para os dias com céu límpido do outono e inverno.

Na Tabela 1, para a superfície $20 \mathrm{~N}$, os desvios acham-se entre $-4,95 \%$ e 5,55\%, sendo o desvio médio igual a $0,54 \%$.

Na Tabela 2, para a superfície 20S, os desvios acham-se entre $-12,88 \%$ e $-4,04 \%$, sendo o desvio médio igual a $-8,05 \%$.

LATANZE et al. (1987), trabalhando com o modelo de KONDRATYEV (1977), constataram que os totais de radiação solar medidos em diferentes orientações e em diferentes épocas do ano apresentaram diferenças inferiores a $2 \%$, comparados com aqueles estimados pelo modelo; resultados diferentes dos obtidos neste experimento, para os períodos avaliados.

Outono

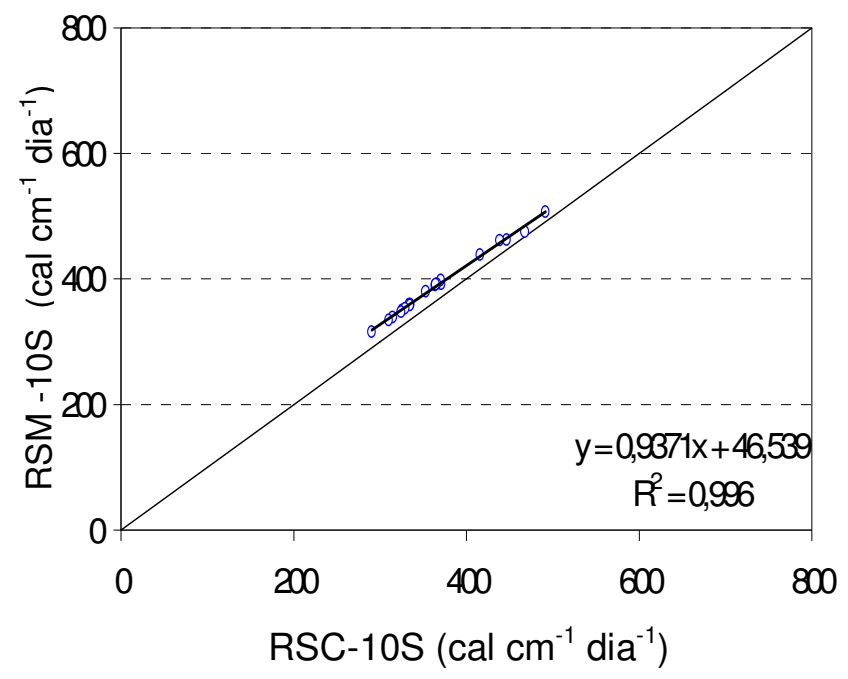

Inverno

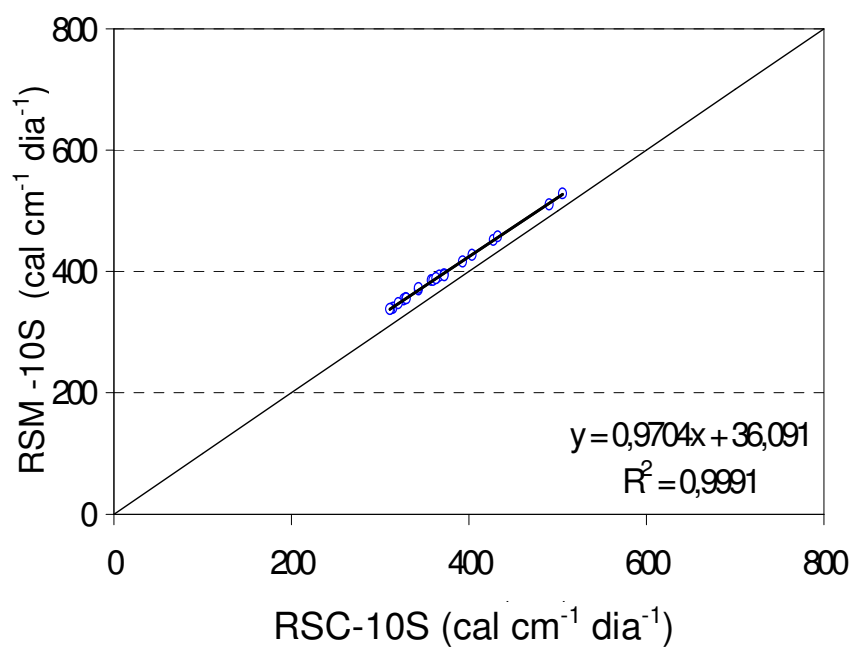

FIGURA 4. Correlação entre os valores diários da radiação solar global calculada em relação à radiação solar medida, na superfície 10S, nos dias com céu límpido do outono e inverno. 

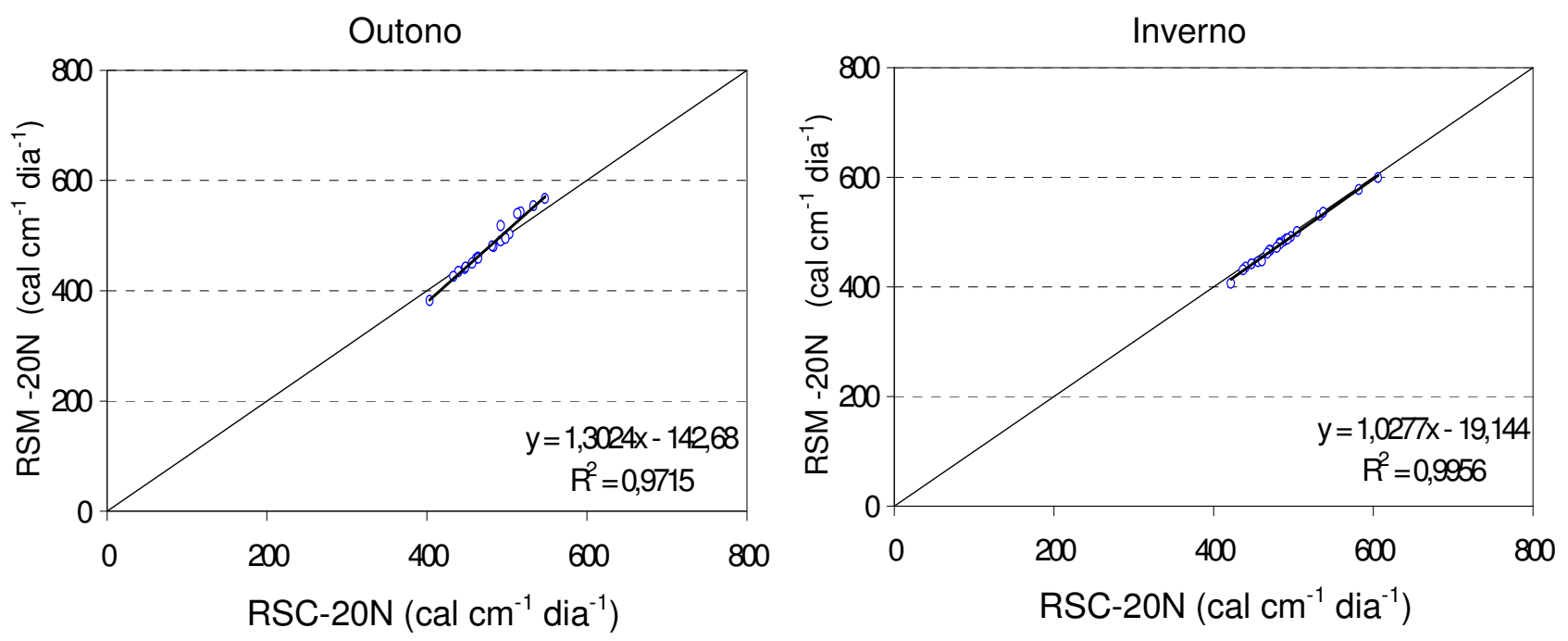

FIGURA 5. Correlação entre os valores diários da radiação solar global calculada em relação à radiação solar medida, na superfície $20 \mathrm{~N}$, nos dias com céu límpido do outono e inverno.
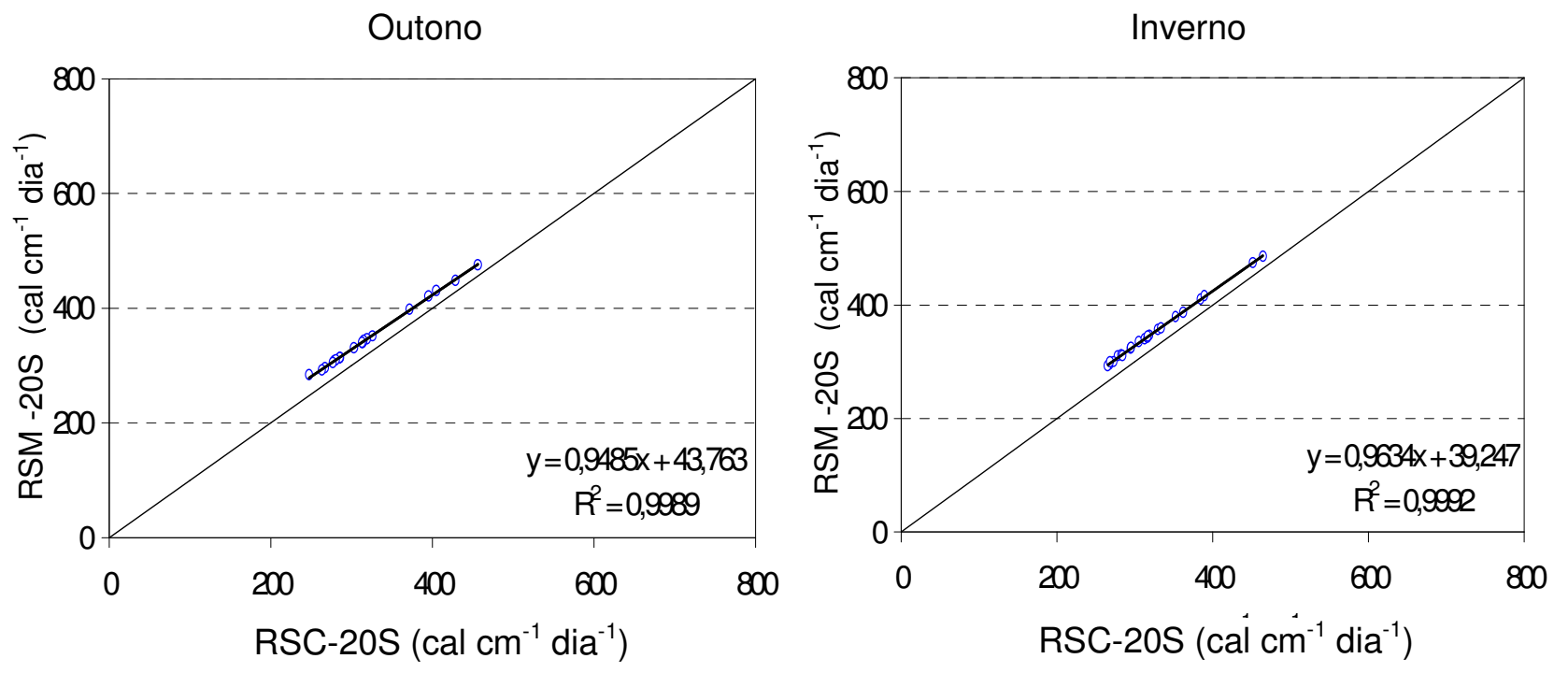

FIGURA 6. Correlação entre os valores diários da radiação solar global calculada em relação à radiação solar medida, na superfície 20 S, nos dias com céu límpido do outono e inverno. 
TABELA 1. Radiação solar global medida e calculada, na superfície 20N, para os dias com céu límpido do outono e inverno.

\begin{tabular}{|c|c|c|c|c|c|}
\hline Superfície & Estação do Ano & Data & $\begin{array}{c}\text { RSM } \\
\left(\text { cal } \mathrm{cm}^{-2} \mathrm{dia}^{-1}\right)\end{array}$ & $\begin{array}{c}\text { RSC } \\
\left(\mathrm{cal} \mathrm{cm}^{-2} \mathrm{dia}^{-1}\right)\end{array}$ & Diferença $(\%)$ \\
\hline \multirow{20}{*}{$20 \mathrm{~N}$} & \multirow{20}{*}{ Outono } & $3-4-2002$ & 567,1499 & 547,7489 & $-3,42$ \\
\hline & & $12-4-2002$ & 554,0302 & 533,2241 & $-3,76$ \\
\hline & & $19-4-2002$ & 542,8075 & 517,4186 & $-4,68$ \\
\hline & & 23-4-2002 & 539,9510 & 513,2299 & $-4,95$ \\
\hline & & $28-4-2002$ & 517,9594 & 492,3747 & $-4,94$ \\
\hline & & $10-5-2002$ & 479,9760 & 483,3855 & 0,71 \\
\hline & & $24-5-2002$ & 490,3397 & 492,4595 & 0,43 \\
\hline & & $25-5-2002$ & 502,8575 & 502,9728 & 0,02 \\
\hline & & $26-5-2002$ & 496,6234 & 497,5481 & 0,19 \\
\hline & & $27-5-2002$ & 494,7806 & 498,3543 & 0,72 \\
\hline & & $28-5-2002$ & 481,5040 & 481,7289 & 0,05 \\
\hline & & $2-6-2002$ & 440,3214 & 447,3963 & 1,61 \\
\hline & & $3-6-2002$ & 458,0108 & 461,4575 & 0,75 \\
\hline & & $4-6-2002$ & 460,6637 & 463,9741 & 0,72 \\
\hline & & $5-6-2002$ & 458,6780 & 464,1144 & 1,19 \\
\hline & & $6-6-2002$ & 382,2497 & 403,4671 & 5,55 \\
\hline & & $7-6-2002$ & 450,1924 & 456,7992 & 1,47 \\
\hline & & $8-6-2002$ & 442,5398 & 448,3798 & 1,32 \\
\hline & & $14-6-2002$ & 434,5882 & 439,4370 & 1,12 \\
\hline & & $15-6-2002$ & 426,0747 & 433,1568 & 1,66 \\
\hline \multirow{22}{*}{$20 \mathrm{~N}$} & \multirow{22}{*}{ Inverno } & $27-6-2002$ & 445,9111 & 455,9137 & 2,24 \\
\hline & & $28-6-2002$ & 436,1173 & 440,2453 & 0,95 \\
\hline & & $1-7-2002$ & 430,8205 & 436,8551 & 1,40 \\
\hline & & $2-7-2002$ & 442,1078 & 447,6101 & 1,24 \\
\hline & & $8-7-2002$ & 491,6955 & 496,8360 & 1,05 \\
\hline & & $14-7-2002$ & 466,9433 & 471,0111 & 0,87 \\
\hline & & $15-7-2002$ & 466,6168 & 470,0044 & 0,73 \\
\hline & & $16-7-2002$ & 447,2283 & 460,6987 & 3,01 \\
\hline & & $17-7-2002$ & 442,0692 & 447,6276 & 1,26 \\
\hline & & $18-7-2002$ & 406,6010 & 421,6346 & 3,70 \\
\hline & & $24-7-2002$ & 480,1602 & 482,9818 & 0,59 \\
\hline & & $25-7-2002$ & 484,3378 & 488,5240 & 0,86 \\
\hline & & $26-7-2002$ & 487,7262 & 490,9732 & 0,67 \\
\hline & & $28-7-2002$ & 478,0524 & 483,0440 & 1,04 \\
\hline & & $8-8-2002$ & 472,0764 & 479,2292 & 1,52 \\
\hline & & $15-8-2002$ & 461,5509 & 467,3470 & 1,26 \\
\hline & & $16-8-2002$ & 487,8011 & 492,9303 & 1,05 \\
\hline & & $17-8-2002$ & 501,0865 & 504,7315 & 0,73 \\
\hline & & $18-8-2002$ & 530,6882 & 533,1727 & 0,47 \\
\hline & & $19-8-2002$ & 536,2503 & 537,5357 & 0,24 \\
\hline & & $2-9-2002$ & 599,1741 & 606,1592 & 1,17 \\
\hline & & $3-9-2002$ & 577,3814 & 581,9539 & 0,79 \\
\hline
\end{tabular}


TABELA 2. Radiação solar global medida e calculada, na superfície 20S para os dias com céu límpido do outono e inverno.

\begin{tabular}{|c|c|c|c|c|c|}
\hline Superfície & Estação do Ano & Data & $\begin{array}{c}\text { RSM } \\
\left(\text { cal } \mathrm{cm}^{-2} \mathrm{dia}^{-1}\right)\end{array}$ & $\begin{array}{c}\text { RSC } \\
\left(\text { cal } \mathrm{cm}^{-2} \mathrm{dia}^{-1}\right)\end{array}$ & Diferença $(\%)$ \\
\hline \multirow{20}{*}{$20 \mathrm{~S}$} & \multirow{20}{*}{ Outono } & $3-4-2002$ & 475,6469 & 456,4488 & $-4,04$ \\
\hline & & $12-4-2002$ & 448,4083 & 428,8636 & $-4,36$ \\
\hline & & $19-4-2002$ & 430,6026 & 405,0303 & $-5,94$ \\
\hline & & 23-4-2002 & 421,0851 & 395,3922 & $-6,10$ \\
\hline & & $28-4-2002$ & 398,1554 & 371,9571 & $-6,58$ \\
\hline & & $10-5-2002$ & 351,6280 & 326,0471 & $-7,27$ \\
\hline & & $24-5-2002$ & 339,5925 & 313,8051 & $-7,59$ \\
\hline & & $25-5-2002$ & 346,8786 & 219,2355 & $-7,97$ \\
\hline & & $26-5-2002$ & 343,6122 & 315,0159 & $-8,32$ \\
\hline & & $27-5-2002$ & 340,4510 & 313,2152 & $-8,00$ \\
\hline & & $28-5-2002$ & 331,1233 & 303,1973 & $-8,43$ \\
\hline & & $2-6-2002$ & 308,4749 & 278,8340 & $-9,61$ \\
\hline & & $3-6-2002$ & 312,9717 & 285,2404 & $-8,86$ \\
\hline & & $4-6-2002$ & 314,2339 & 286,0027 & $-8,98$ \\
\hline & & $5-6-2002$ & 314,0563 & 285,6379 & $-9,05$ \\
\hline & & $6-6-2002$ & 284,2052 & 247,6012 & $-12,88$ \\
\hline & & $7-6-2002$ & 309,7581 & 280,7490 & $-9,37$ \\
\hline & & $8-6-2002$ & 305,5120 & 277,0580 & $-9,31$ \\
\hline & & $14-6-2002$ & 296,0871 & 267,3041 & $-9,72$ \\
\hline & & $15-6-2002$ & 292,1737 & 263,5305 & $-9,80$ \\
\hline \multirow{22}{*}{$20 \mathrm{~S}$} & \multirow{22}{*}{ Inverno } & $27-6-2002$ & 310,3441 & 278,2161 & $-10,35$ \\
\hline & & $28-6-2002$ & 295,2926 & 267,6769 & $-9,35$ \\
\hline & & $1^{\mathrm{o}}-7-2002$ & 292,8656 & 265,4328 & $-9,37$ \\
\hline & & $2-7-2002$ & 300,1589 & 272,8499 & $-9,10$ \\
\hline & & $8-7-2002$ & 335,5555 & 305,2200 & $-9,04$ \\
\hline & & $14-7-2002$ & 323,6134 & 294,9621 & $-8,85$ \\
\hline & & $15-7-2002$ & 325,3165 & 295,2509 & $-9,24$ \\
\hline & & $16-7-2002$ & 312,0645 & 282,9190 & $-9,34$ \\
\hline & & $17-7-2002$ & 310,9036 & 284,2473 & $-8,57$ \\
\hline & & $18-7-2002$ & 299,8582 & 268,5053 & $-10,46$ \\
\hline & & $24-7-2002$ & 340,4251 & 312,7525 & $-8,13$ \\
\hline & & $25-7-2002$ & 342,6082 & 316,1166 & $-7,73$ \\
\hline & & $26-7-2002$ & 346,8538 & 319,0873 & $-8,01$ \\
\hline & & $28-7-2002$ & 345,3483 & 316,7166 & $-8,29$ \\
\hline & & $8-8-2002$ & 356,9991 & 329,9514 & $-7,58$ \\
\hline & & $15-8-2002$ & 359,4017 & 333,8046 & $-7,12$ \\
\hline & & $16-8-2002$ & 379,2031 & 352,5478 & $-7,03$ \\
\hline & & $17-8-2002$ & 387,0486 & 362,4463 & $-6,36$ \\
\hline & & $18-8-2002$ & 410,3905 & 384,5453 & $-6,30$ \\
\hline & & $19-8-2002$ & 416,2952 & 389,5465 & $-6,43$ \\
\hline & & $2-9-2002$ & 485,7785 & 464,6505 & $-4,35$ \\
\hline & & $3-9-2002$ & 474,4009 & 451,7314 & $-4,78$ \\
\hline
\end{tabular}




\section{CONCLUSÕES}

O modelo estudado foi preciso para estimar a radiação solar nas superfícies $\mathrm{H}, 10 \mathrm{~N}, 10 \mathrm{E}, 10 \mathrm{~W}$, $20 \mathrm{E}$ e $20 \mathrm{~W}$, para as quatro estações do ano, e para as superfícies $10 \mathrm{~S}, 20 \mathrm{~N}$ e $20 \mathrm{~S}$, para a primavera e o verão. Porém, na superfície $10 \mathrm{~S}$, para o outono e o inverno, e nas superfícies $20 \mathrm{~N}$ e $20 \mathrm{~S}$, para o outono, o modelo apresenta resultados aceitáveis. Já para o inverno, o modelo não apresentou bons resultados para estimar a radiação nas superfícies $20 \mathrm{~N}$ e $20 \mathrm{~S}$.

Utilizando-se de dados de dias com céu límpido, constatou-se que, no inverno, o modelo foi preciso para estimar a radiação solar na superfície $20 \mathrm{~N}$ e apresentou resultados aceitáveis para estimar a radiação solar na superfície 20 S.

\section{REFERÊNCIAS}

ARAGON, F.F.; TOLEDO PIZA, J.A.N. Modelo matemático generalizado de radiação solar diária sobre superfícies planas. Energia na Agricultura, Botucatu, v.6, n.1, p.5-14, 1991.

CHANG, J.H. Climate and agriculture - an ecological survey. Chicago: Aldine Publishing Company, 1968. p.87-99.

COLLE, S; PEREIRA, E.B. Atlas de irradiação solar do Brasil. Brasília: LABSOLAR, 1998. 65 p.

KANG, S; KIM, S; LEE, D. Spatial and temporal patterns of solar radiation based on topography and air temperature. Canadian Journal of Forest Research, Ottawa, v.32, n.3, p.487-97, 2002.

KONDRATYEV, K.Y. Radiation regime of inclined surfaces. Genebra; World Meteorological Organization, 1977. 82 p. (Technical note, 152).

KONDRATYEV, K.Y.; MANOLOVA, M.P. The radiation balance of slopes. Solar Energy, Kidlinton, v.4, n.1, p.14-19, 1960.

LATANZE, R.J.; BENINCASA, M.; LOPES, L.R. Estimativa da radiação solar incidente sobre superfícies com diferentes orientações. In: CONGRESSO BRASILEIRO DE ENGENHARIA AGRÍCOLA, 16., 1987, Jundiaí. Anais... Jundiaí: Sociedade Brasileira de Engenharia Agrícola, 1987. p.646-7.

LOPES, L.R. Efeitos da topografia sobre a variação da radiação solar incidente, temperatura do solo e produção de Cynodon dactylon (L.) Pers cv. Coastcross I, 1986. 103 f. Tese (Livre-Docência) Faculdade de Ciências Agrárias e Veterinárias, Universidade Estadual Paulista, Jaboticabal, 1986.

VIENELLO, RL.; ALVES, A.R. Meteorologia básica e aplicações. Viçosa: Imprensa Universitária, 1991. p.133-200.

WASSINK, E.C. Light energy conversion in photosynthesis and growth of plants. Copenhagem: UNESCO, 1968. p.53-66. 